DEVELOPMENTAL BIOLOGY

\section{Early embryos kept in check}

As pluripotent stem cells become primed to give rise to all bodily cell types, they begin to form the amniotic cavity in which the mammalian fetus will grow. A checkpoint that gates this transition has now been identified.

\section{JULIEN G. DUMORTIER \& JEAN-LÉON MAÎTRE}

$\mathrm{D}$ uring embryonic development, cells differentiate into particular lineages according to information about their position in the embryo. This adoption of a particular cellular identity often triggers changes in the organization of the embryo, which, in turn, results in new positional information. These cycles of differentiation and morphogenesis are key to normal development - but how do embryos coordinate them? One way is to implement checkpoints, similar to those in place during the cell cycle, to block progression until specific criteria are met. In a paper online in Nature, Shahbazi et al. ${ }^{1}$ report just such a checkpoint during early development in mammals.

In mammalian development, the embryo forms a structure called the blastocyst ${ }^{2}$, which contains a mass of pluripotent cells - those that can give rise to any cell type in the body. In most mammals, the blastocyst implants into maternal hormones, which induce the blastocyst to secrete the protein leukaemia inhibitory factor (LIF) and to enter a dormant state ${ }^{3}$.

Pluripotent cells isolated from the blastocyst for growth in vitro - called embryonic stem (ES) cells - can be maintained in a 'naive' pluripotent state by LIF (ref. 4). When LIF is removed, ES cells become 'primed' and can initiate their differentiation into the various progenitors that build the body. The role of these different states in vivo is not clear, but one hypothesis has been that LIF removal primes the embryo to resume development ${ }^{3}$, thereby constituting a bona fide checkpoint for implantation.

At the implantation stage of development, embryos also undergo a dramatic change in shape. The amniotic cavity, which will contain the growing embryo and the fluids that surround it, begins to form within a circle of primed pluripotent cells. The formation of the amniotic cavity has been poorly characterized, because this process occurs during early stages the uterus. Implantation can be put on hold by of embryo implantation, which are difficult to access experimentally. But key insights into the process have come in the past three years, thanks to previous work from the group that performed the current study. The authors, along with other groups, greatly improved in vitro culture conditions for human ${ }^{5,6}$ and mouse ${ }^{7}$ embryos, and succeeded in growing embryos outside the uterus until shortly after the implantation stage.

Shahbazi et al. used their ex utero culture systems to study the coordination between the exit from pluripotency and the formation of the amniotic cavity. First, the authors noticed that the downregulation of pluripotency genes coincided with the appearance of the amniotic cavity in both mouse and human embryos. Next, they observed that maintaining pluripotent cells in a naive state in embryos by artificially supplying LIF prevented the cells from forming the amniotic cavity.
To carefully dissect this coordinated process, Shahbazi and colleagues turned to mouse ES cells as a proxy for the embryo. When placed in specific culture conditions, ES cells can cohesive tissue in which polarized cells align along their apical-basal axes and form specialized contacts called tight junctions. And, like other epithelial cells ${ }^{8}$, ES cells can form a structure resembling the amniotic cavity when grown in $3 \mathrm{D}$ culture systems ${ }^{5,7}$. However, the authors found that the cells do so only when primed by the removal of LIF. They therefore investigated the specific step that is controlled by LIF removal.

Shahbazi et al. determined that naive ES cells grown in 3D culture undergo several of the steps needed to form a cavity. First, the cells arrange into circular rosette structures, with their apical ends in close proximity (Fig. 1). Second, fluid-containing vesicles are trafficked towards the apical interfaces between cells, and tight junctions form. These steps are key prerequisites for the accumulation of fluid between apical interfaces ${ }^{9}$. However, the authors found that naive cells lack negatively charged, membrane-spanning apical proteins called sialomucins, which induce the repulsion of contacting apical membranes to form a negatively charged gap in the centre of the rosette $^{10}$. Shahbazi and colleagues showed that sialomucin production is induced after LIF removal, suggesting that the checkpoint is not the formation of a polarized epithelium, but the repulsion of apical surfaces. form an epithelium - a highly organized and
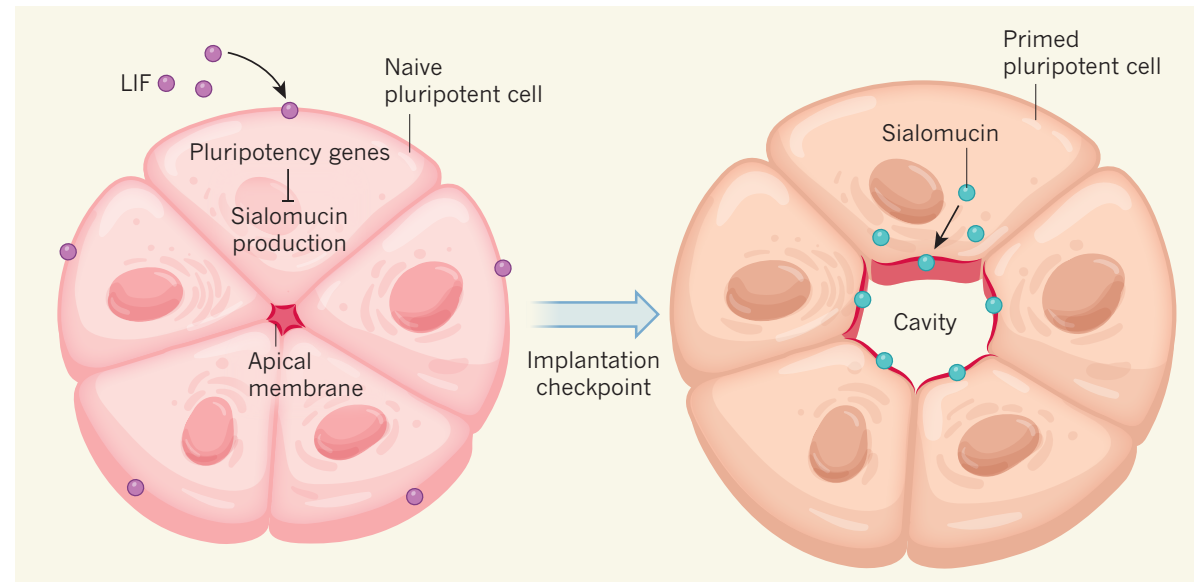

Figure 1 | A checkpoint that coordinates embryonic development. As mouse and human embryos implant into the uterus, the amniotic cavity (which will hold the developing fetus) begins to form. Shahbazi et al. ${ }^{1}$ provide in vitro evidence that, before implantation, naive pluripotent cells (which can give rise to all bodily cell lineages) place their apical edges in contact to form a polarized 'rosette' structure. The secreted protein leukaemia inhibitory factor (LIF) promotes expression of pluripotency genes in naive pluripotent cells, and these genes, in turn, inhibit the production of sialomucins - negatively charged proteins needed for amniotic-cavity formation. During implantation, LIF is removed and pluripotent cells become primed to differentiate. At this stage, the polarized transport of sialomucins towards apical interfaces causes them to repel each other, leading to cavity opening. This set-up ensures that implantation of the mammalian embryo is coordinated with its development. 
Next, the researchers used available genomic data ${ }^{11}$ to gain mechanistic insight into how the exit from naive pluripotency controls cavity formation. This analysis unveiled a potential role for the protein OCT4 in regulating sialomucin expression. When taken together with a recent study ${ }^{12}$ that described the effects of OCT4 deficiency on preimplantation human embryos, the authors' finding suggests an important role for OCT4 in controlling early human development.

Although the expression of sialomucins is essential for cavity formation, the group found that addition of these proteins to naive cells did not, in itself, induce such a transition. This suggests that the delivery of sialomucins to the apical interface might require other proteins, such as the tight-junction protein cingulin, which the researchers showed was produced upon LIF removal. Cingulin has been implicated in the tethering of vesicles to the apical membrane, indicating that it might have a role in cavity formation ${ }^{13}$.

Future work will be needed to identify the cocktail of proteins that is sufficient to trigger naive cells to form a cavity. Moreover, whether the molecular events described in ES cells occur in mouse and human embryos remains to be verified. Understanding how the formation of the amniotic cavity is regulated at the molecular and cellular levels will support future studies of the physical events that control cavity formation, and will lay the foundations for modelling this process ${ }^{2}$.

Altogether, Shahbazi and colleagues' findings allow them to outline a mechanism by which embryos coordinate the exit from pluripotency and the formation of the amniotic cavity. In this scenario, naive cells form a polarized epithelium that is poised for cavitation. Only when cells become primed do their apical interfaces begin to repel one another, allowing fluid to accumulate. This provides evidence that the naive state has a biological role, acting as a checkpoint to ensure that the amniotic cavity forms only when cells are primed. In this way, embryos can keep morphogenesis and differentiation in synchrony.
Julien G. Dumortier and Jean-Léon Maître are in the Unité Génétique et Biologie du Développement, Institut Curie, PSL Research University, CNRS UMR3215, INSERM U934, 75248 Paris, France.

e-mail:jean-leon.maitre@curie.fr

1. Shahbazi, M. N. et al. Nature http://dx.doi. org/10.1038/nature24675 (2017).

2. Maître, J.-L. Biol. Cell 109, 323-338 (2017).

3. Frankenberg, S. R., de Barros, F. R. O., Rossant, J. \& Renfree, M. B. WIREs Dev. Biol. 5, 210-232 (2016).

4. Ying, Q.-L. et al. Nature 453, 519-523 (2008)

5. Shahbazi, M. N. et al. Nature Cell Biol. 18, $700-708$ (2016).

6. Deglincerti, A. et al. Nature 533, 251-254 (2016)

7. Bedzhov, I. \& Zernicka-Goetz, M. Cell 156, 1032-1044 (2014).

8. Bryant, D. M. et al. Nature Cell Biol. 12, 1035-1045 (2010).

9. Blasky, A. J., Mangan, A. \& Prekeris, R. Annu. Rev. Cell Dev. Biol. 31, 575-591 (2015).

10.Strilić, B. et al. Curr. Biol. 20, 2003-2009 (2010).

11.Buecker, C. et al. Cell Stem Cell 14, 838-853 (2014).

12.Fogarty, N. M. E. et al. Nature 550, 67-73 (2017).

13.Mangan, A. J. et al. Nature Commun. 7, 12426 (2016). 